\title{
PREPARAÇÃO, CARACTERIZAÇÃO FÍSICO-QUÍMICA E LIBERAÇÃO CONTROLADA DE MICROPARTÍCULAS DE GALACTOMANANA CONTENDO ALANTOÍNA
}

\author{
Hilana B. M. Frota ${ }^{a}$, Jane Eire S. A. Menezes ${ }^{a}$, Sônia Maria C. Siqueira ${ }^{a}$, Nágila Maria P. Ricardo ${ }^{b}$, Tamara G. Araújo \\ Carlos Alberto G. Souza ${ }^{d}$, Paulo N. Bandeira ${ }^{\mathrm{e}}$ e Hélcio S. dos Santos ${ }^{\mathrm{e}, *}$ \\ aDepartamento de Química, Universidade Estadual do Ceará, 60740-903 Fortaleza - CE, Brasil \\ bDepartamento de Química Orgânica e Inorgânica, Universidade Federal do Ceará, 60455-760 Fortaleza - CE, Brasil \\ 'Departamento de Farmácia, Universidade Federal do Ceará, 60430-160 Fortaleza - CE, Brasil \\ ¿Departamento de Química, Universidade Federal da Bahia, 40170-115 Salvador - BA, Brasil \\ eDepartamento de Química, Universidade Estadual Vale do Acaraú, 62040-370 Sobral - CE, Brasil
}

Recebido em 06/01/2018; aceito em 22/02/2018; publicado na web em 08/03/2018

\begin{abstract}
PREPARATION, PHYSICOCHEMICAL CHARACTERIZATION AND CONTROLLED RELEASE OF GALACTOMANNAN MICROPARTICLES CONTAINING ALLANTOIN. In this work, the allantoin was encapsulated with galactomannan extruded from Delonyx regia, by the spray drying technique. The microparticles obtained were structurally characterized by infrared spectroscopy (IR), thermal analysis, scanning electron microscopy (SEM) and the controlled release profile was evaluated. The results of IR suggested the formation of the polymeric wall of the microcapsules and SEM revealed spherical particles with an average size range of $2.84 \pm 0.41 \mu \mathrm{m}$. X-ray diffraction showed the loss of crystallinity of the active after encapsulation and differential explanatory calorimetry exhibited the loss of its peak melt. The encapsulation efficiency for the microparticles was approximately $84 \%$ for the different environmental conditions. The coating of allantoin with the biopolymer promoted a gradual release of the drug over a period of 6 hours and these results are in accordance with the Brazilian Pharmacopoeia. The set of results obtained in this work is relevant and accredits the system developed as a possible alternative for the formation of encapsulated natural drugs destined to possible treatments of cutaneous cellular regeneration.
\end{abstract}

Keywords: galactomannan; allantoin; microencapsulation; characterization; controlled release.

\section{INTRODUÇÃO}

Polissacarídeos fazem parte do nosso cotidiano e representam uma das classes de materiais mais versáteis, destacando-se principalmente nas mais diversas áreas como medicina, materiais e alimentos. ${ }^{1}$ No setor farmacêutico, polímeros podem ser utilizados como moduladores e direcionadores da liberação de fármacos em sítios específicos no organismo. O que vem ser de grande importância, uma vez que medicamentos tradicionais são caracterizados por apresentarem liberação imediata do fármaco. ${ }^{2,3}$ Por outro lado, a liberação controlada de fármacos promovida pela encapsulação dos compostos ativos em micropartículas carreadoras tem se tornado uma ferramenta bastante útil no sentido de prolongar o tempo de liberação do fármaco no organismo. ${ }^{4}$

A pele é considerada o maior órgão do corpo humano, sendo o principal sítio de interação com o ambiente externo. ${ }^{5}$ Além disso, a pele serve como uma barreira protetora, sendo capaz de prevenir ou minimizar a exposição dos tecidos a eventos traumáticos, radiação ultravioleta, variações de temperatura, toxinas e contato direto com microorganismos. ${ }^{6}$

Feridas cutâneas são caracterizadas pelo rompimento da estrutura e função dos tecidos subjacentes. ${ }^{7}$ As feridas podem ser classificadas como agudas ou crônicas, dependendo de sua tendência para o processo de cicatrização. ${ }^{8}$ A cicatrização é descrita como um processo complexo onde múltiplas vias, de eventos fisiopatológicos e bioquímicos que ocorrem em paralelo e de forma inter-relacionada, são ativadas e sincronizadas para induzir o reparo tecidual. ${ }^{9}$

Aproximadamente um terço de todos os medicamentos derivados de plantas é utilizado para o tratamento de feridas cutâneas ou outras

*e-mail: helciodossantos@gmail.com doenças de pele, quando comparado a medicamentos convencionais de uso clínico. ${ }^{10} \mathrm{O}$ confrei (Symphytum oficinale) é uma planta de origem europeia e asiática utilizada para no tratamento de lesões cutâneas abertas decorrentes de queimaduras, fraturas e cicatrização de feridas. As partes mais usadas do confrei, cujas propriedades terapêuticas estão relacionadas a presença da alantoína são as folhas e raízes. A alantoína (Figura 1) é um pó branco, cristalino, de origem orgânica, que favorece a proliferação celular acelerando a epitalização em zonas lesadas da pele ou submetidas a um grande desgaste, ou a intempéries do tempo. ${ }^{11}$<smiles>NC(=O)NC1NC(=O)NC1=O</smiles>

Figura 1. Representação estrutural da alantoína (2,5-dioxo-4-imidazolidiniluréia)

Desta forma, o desenvolvimento de formulações farmacêuticas envolvendo compostos bioativos encapsulados em matrizes poliméricas, objetivando a liberação controlada de fármacos, representa uma nova estratégia para incorporação de substâncias ativas e maior controle da liberação deste princípio ativo. Além disso, reduz efeitos colaterais indesejados uma vez que utiliza uma menor quantidade do princípio ativo, resultando em menor custo. ${ }^{12}$ Dentre as estruturas carreadoras empregadas, incluem-se as micropartículas, as quais são consideradas Sistemas de Liberação de Fármacos (SLF), formadas pela combinação polímero-fármaco, em que o fármaco se encontra homogeneamente disperso na matriz polimérica. Esses sistemas 
possuem algumas vantagens sobre os sistemas implantáveis, pois, devido ao seu tamanho, podem ser colocados diretamente no local desejado, atuando como um reservatório do fármaco e promovendo a sua lenta liberação. ${ }^{13}$

Assim, o principal objetivo do presente trabalho foi investigar a capacidade da galactomanana obtida de $D$. régia para encapsular a alantoína pela técnica de Spray-drying, realizar a caracterização fisico-química por meio de técnicas espectroscópicas (RMN e IV), termo analíticas (TGA e DSC), Microscopia eletrônica de varredura (MEV), raios X, além de verificar a liberação controlada da alantoína encapsulada nas micropartículas de galactomanana.

\section{MATERIAL E MÉTODOS}

\section{Material vegetal}

As sementes de Delonix regia (Hook.) Raf., Leguminosae, foram coletadas entre os meses de março a junho de 2015 no Campus da Universidade Estadual do Ceará, no Município de Fortaleza-CE. Uma exsicata da espécie encontra-se depositada no Herbário Prisco Bezerra, do Departamento de Biologia da Universidade Federal do Ceará, sob número de registro 53.140.

\section{Materiais}

Foram utilizados alantoína $\left(\mathrm{C}_{4} \mathrm{H}_{6} \mathrm{~N}_{4} \mathrm{O}_{3} ; 158,121 \mathrm{~g} \mathrm{~mol}^{-1}\right)$ adquirida na Sigma - Brasil, e álcool etílico anidro 99,3 INPM (99,5 $\left.{ }^{\circ} \mathrm{GL}\right)$ na VETEC - Brasil.

\section{Extração da galactomanana}

As galactomananas foram obtidas a partir do endosperma de 200 g de sementes por extração aquosa, seguido por uma precipitação com etanol. ${ }^{14}$

\section{Preparação das micropartículas}

Para a preparação das micropartículas, 3,2 g de galactomanana atomizada foram pesadas e dissolvida em $800 \mathrm{~mL}$ de água destilada em agitação constante ( $700 \mathrm{rpm}$ ) e aquecimento de $40^{\circ} \mathrm{C}$ por 24 horas. Após decorrer este período, foram adicionados $320 \mathrm{mg}$ de alantoína em agitação por 1 hora. Logo após, a solução foi atomizada em aparelho spray dryer Buchi-290 do Laboratório de Polímeros Naturais - LABPIN da Universidade Federal do Ceará, usando temperatura de entrada de $120^{\circ} \mathrm{C}$; temperatura de saída de $90{ }^{\circ} \mathrm{C}$; eficiência do exaustor de $90 \%$ e fluxo de bomba de $30 \%$.

\section{Eficiência de Encapsulamento}

\section{Curva Padrão}

Para a construção da curva padrão analítica, foi preparada uma solução estoque aquosa de alantoína em uma concentração de 200 $\mu \mathrm{g} \mathrm{mL}{ }^{-1}$. Diferentes alíquotas desta solução foram transferidas para balões volumétricos de $10 \mathrm{~mL}$ e o volume completado com água destilada a fim de obter soluções de diferentes concentrações (40, $35,30,25,20,15,10$ e $\left.5 \mu \mathrm{gL}^{-1}\right)$. A absorbância das soluções foi determinada por espectrometria na região UV-Vis em $194 \mathrm{~nm}$, usando água destilada como branco. Com a curva de calibração obtivemos a equação 1 de regressão linear entre os valores das absorbâncias e a concentração da alantoina em $\mu \mathrm{g} \mathrm{ml}^{-1}$.

$$
Y=0,034 x+0,058, \text { onde } R^{2}=0,986
$$

Quantificação de alantoína nas micropartículas por espectrofotometria $U V$-Vis

Foram separadas $30 \mathrm{mg}$ de micropartículas para diferentes condições ambientais [temperatura $25^{\circ} \mathrm{C}, 60{ }^{\circ} \mathrm{C}$ e sob ação da luz $(\lambda)]$ por sete dias. Após decorrer este tempo, para cada amostra das determinadas condições, foram dissolvidos $2 \mathrm{mg} \mathrm{em} 10 \mathrm{~mL}$ de água destilada para obter soluções de $200 \mu \mathrm{g} \mathrm{mL}^{-1}$ em 10 frascos diferentes, os quais foram analisadas em espectrômetro na região UV-Vis a $194 \mathrm{~nm}$. A concentração do fármaco foi determinada usando a curva padrão e a eficiência de encapsulamento foi calculada de acordo com a equação 2 :

$$
E E(\%)=[Q T D / Q T A] \times 100
$$

sendo $\mathrm{EE}=$ eficiência de encapsulação; $\mathrm{QTD}=$ quantidade de fármaco determinado no sistema polimérico; QTA= quantidade de fármaco teoricamente adicionado no sistema polimérico.

Para calcular o teor de alantoina encapsulada foi utilizada a equação 3:

$$
T A(\%)=[(T T A \times E E) / 100]
$$

sendo TA $(\%)=$ teor de alantoina encapsulada; TTA $(\%)=$ teor teórico de alantoina encapsulada; $\mathrm{EE}=$ eficiência de encapsulação.

\section{Caracterização da galactomanana}

Análise por Ressonância Magnética Nuclear do hidrogênio $\left(R M N^{1} H\right)$

Foram preparadas soluções das amostras da galactomanana ( $D$. regia) em $\mathrm{D}_{2} \mathrm{O}$ na concentração de $27 \mathrm{mg} 600 \mu \mathrm{L}^{-1}$, sendo as amostras deixadas em $\mathrm{D}_{2} \mathrm{O}$ nessa concentração por 24 horas sob agitação constante e a temperatura de $80{ }^{\circ} \mathrm{C}$. Os espectros de ressonância magnética nuclear do hidrogênio $\left(\mathrm{RMN}^{1} \mathrm{H}\right)$ foram obtidos por meio do espectrômetro Bruker Spectrometers, modelo Avance DRX-500, com transformada de Fourier, no Centro Nordestino de Aplicação e Uso da Ressonância Magnética Nuclear (CENAUREMN), operando na frequência do hidrogênio a $500 \mathrm{MHz}$ e utilizando uma sonda dual de $5 \mathrm{~mm}$. Os espectros foram registrados à temperatura de $80{ }^{\circ} \mathrm{C}$. Foi usado como padrão interno nas amostras o mesmo solvente de sua diluição $\left(\mathrm{D}_{2} \mathrm{O}\right)$. Para os espectros de $\mathrm{RMN}{ }^{1} \mathrm{H}$, são integrados os sinais dos hidrogênios pertencentes aos carbonos anoméricos da amostra. Através das integrações desses dois sinais, em torno de 5,19 e 5,47 ppm, foi calculada a razão manose:galactose (M:G) da galactomanana da $D$. regia.

\section{Espectroscopia de absorção na região do infravermelho}

Os espectros de absorção na região do infravermelho (IV) foram obtidos utilizando um espectrofotômetro modelo FTIR Spectrometer Frontier, marca Perkin Elmer. Foi utilizado um acessório de reflectância total (ATR) atenuada com um cristal de seleneto de zinco (ZnSe). As amostras foram trituradas com brometo de potássio de grau espectroscópico $(\mathrm{KBr})$ em pó para obtenção das pastilhas. A medição IV foi feita no intervalo de números de onda de 500-4.000 $\mathrm{cm}^{-1}$, resolução de $4 \mathrm{~cm}^{-1}$ e varredura de 16 scans, na Central Analítica da Universidade Federal da Bahia.

\section{Morfologia das micropartículas}

A morfologia das amostras de alantoína, galactomanana e micropartículas de galactomanana/alantoína foram visualizadas nas micrografias produzidas pelo Microscópio Eletrônico de Varredura (MEV), modelo Zeiss DSM 940A operando a $15 \mathrm{kV}$. As amostras foram preparadas em stubs, camada metálica de platina $(60 \mathrm{~nm}$ de 
espessura) no evaporador EMS, na Central Analítica da Universidade Federal do Ceará.

\section{Análises térmicas}

As propriedades térmicas das amostras de alantoína, galactomanana e micropartículas de galactomanana/alantoína foram determinadas usando Calorimetria Diferencial de Varredura (DSC) e análise termogravimétrica (TGA). As curvas de DSC foram realizadas em um equipamento Shimadzu modelo DSC 50, utilizando cadinho de platina e atmosfera de nitrogênio, com uma vazão de $50 \mathrm{~mL} \mathrm{~min}{ }^{-1}$ no intervalo de temperatura de $25-500{ }^{\circ} \mathrm{C}$, a uma taxa de aquecimento de $10{ }^{\circ} \mathrm{C} \mathrm{min}^{-1} \mathrm{e}$ a massa das amostras foi de $5 \mathrm{mg}$. As curvas termogravimétricas das amostras foram obtidas por um equipamento Shimadzu modelo TGA 50, usando um cadinho de platina, atmosfera de ar sintético, com $50 \mathrm{~mL} \mathrm{~min}^{-1}$, entre as bandas de temperatura de 25-800 ${ }^{\circ} \mathrm{C}$, com taxas de aquecimento de $10{ }^{\circ} \mathrm{C} \mathrm{min}^{-1}$, e a massa inicial das amostras analisadas foi de $10 \mathrm{mg}$. Estas análises foram realizadas no Laboratório de análises térmicas da Universidade Federal do Ceará.

\section{Difração de Raios-X}

As amostras de alantoína, galactomanana e micropartículas de galactomanana/alantoína foram analisadas em difratômetro Panalytical, Xpert Pro MPD do Departamento de Física da Universidade Federal do Ceará, utilizando radiação $\operatorname{CoK} \alpha$, uma tensão de $40 \mathrm{kV}$ e uma corrente de $40 \mathrm{~mA}$. A taxa de varredura empregada foi de $0,5^{\circ} \mathrm{min}^{-1}$ em região de $3^{\circ}$ a $90^{\circ}$ de ângulo de difração (20). As amostras foram trituradas para obtenção de um pó fino antes da análise.

\section{Ensaio de liberação in vitro com dispersão direta no meio}

Cerca de $10 \mathrm{mg}$ de alantoína e $120 \mathrm{mg}$ de micropartciculas de galactomanana/alantoína exatamente pesados, foram dispersos em $250 \mathrm{~mL}$ de água destilada a $37 \pm 0,5^{\circ} \mathrm{C}$, sob condições sink, durante 6 horas, utilizando dissolutor (Erweka, modelo DT 800), com aparato II (pá), velocidade de agitação de $50 \mathrm{rpm}$. A metodologia foi adaptada a partir do método descrito pela USP (2007) para cápsulas de liberação prolongada de teofilina (teste 3 ). Foram retiradas alíquotas de $5 \mathrm{~mL}$ do meio de dissolução em tempos previamente determi$\operatorname{nados}(15,30,60,120,180,240,300$ e 360 minutos). As amostras foram diluídas em água destilada e as leituras foram realizadas em espectrofotômetro Genesys, modelo 10 na região UV-Vis a 194 nm. $\mathrm{O}$ ensaio foi realizado em triplicata. ${ }^{15}$

\section{RESULTADOS E DISCUSSÃO}

\section{Caracterização da galactomanana}

A proporção de D-manose e D-galactose foi estimada em 4:1 pela medida direta das áreas relativas aos sinais de ${ }^{1} \mathrm{H}$ (Man) e ${ }^{1} \mathrm{H}(\mathrm{Gal})$. Este resultado está de acordo com trabalhos anteriores para outras galactomananas, incluindo a goma da D. regia.${ }^{16} \mathrm{Com}$ base no assinalamento dos sinais de hidrogênios anoméricos foram identificados os sinais de H de $\alpha$-D-manose (5,26 ppm) e de $\beta$-D-galactose (5,54 ppm) (Figuras 2 e 3 ).

\section{Eficiência de encapsulamento}

De acordo com os resultados observa-se que a eficiência de encapsulamento da alantoína com a galactomanana foi na proporção 10:1 (galactomanana:alantoína) e o teor de alantoína incorporado ao sistema polimérico em diferentes condições (Tabela 1). Os referidos dados estão de acordo com os de Carlos Souza e colaboradores quando relataram que a microencapsulação do ácido L-ascórbico

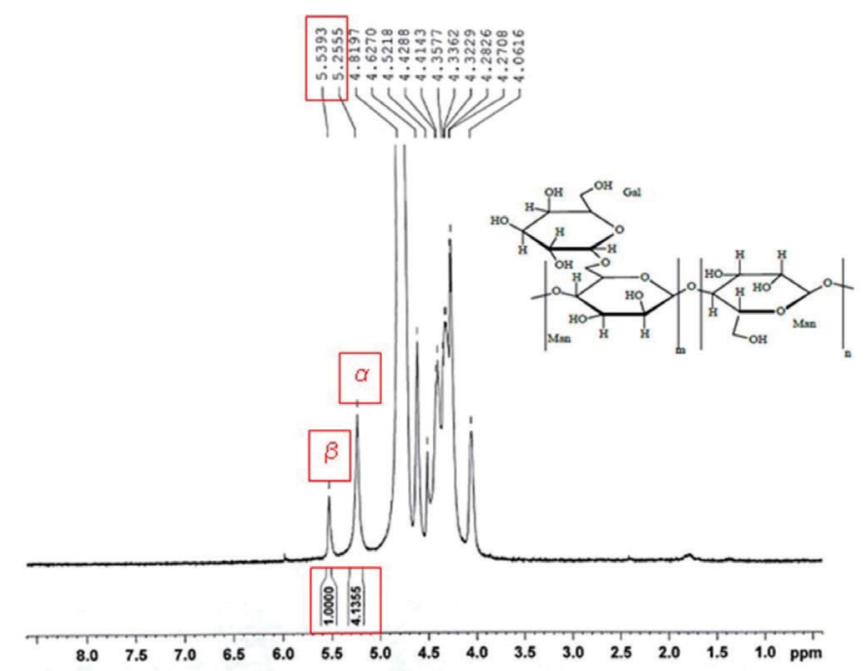

Figura 2. Espectro de $R M N^{1} H$ da galactomanana de D. regia

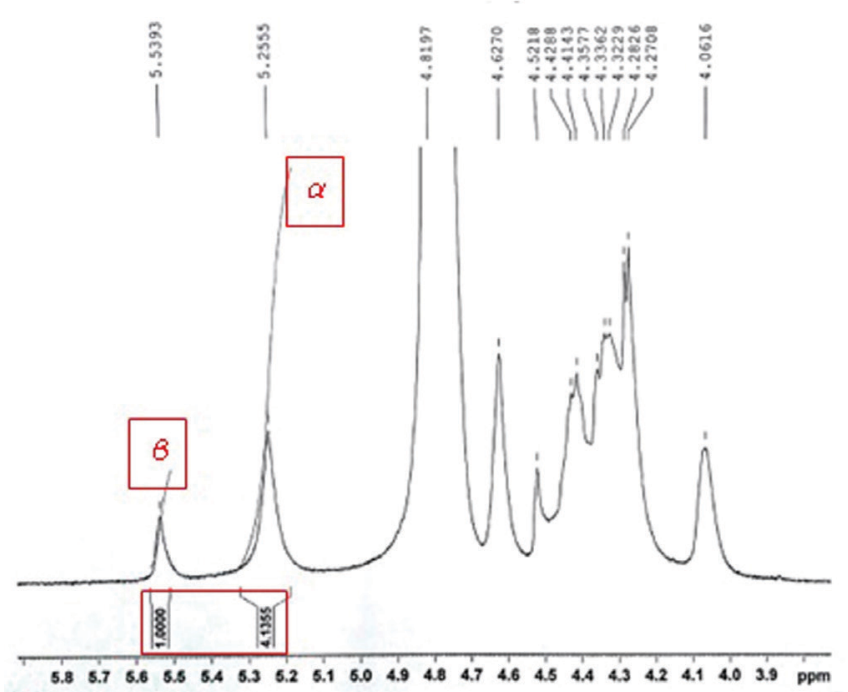

Figura 3. Expansão do espectro de $R M N^{1} \mathrm{H}$ da galactomanana de D. regia

Tabela 1. Eficiência de encapsulação das micropartículas contendo alantoína

\begin{tabular}{lccc}
\hline Amostra & TTA $(\%)$ & EE $(\%)$ & TIA $(\%)$ \\
\hline MGA $\left(\mathrm{T}=25^{\circ} \mathrm{C}\right)$ & 10 & $84,10 \pm 2,26$ & $8,41 \pm 0,23$ \\
$\operatorname{MGA}\left(\mathrm{T}=60^{\circ} \mathrm{C}\right)$ & 10 & $82,00 \pm 3,26$ & $8,20 \pm 0,32$ \\
$\operatorname{MGA}(\lambda)$ & 10 & $84,85 \pm 1,08$ & $8,48 \pm 0,11$ \\
\hline
\end{tabular}

Legenda: MGA - Micropartículas galactomana/alantoína, T - temperatura, $\lambda$ - presença de luz, TTA - teor teórico alantoína, TIA - teor incorporado de alantoína e EE- eficiência de encapsulação.

utilizando galactomanana como agente encapsulante foi de aproximadamente $96 \% .^{16}$

Espectroscopia de absorção na região do Infravermelho (IV)

A espectroscopia na região do infravermelho foi realizada a fim de verificar possíveis interações entre o fármaco e os componentes da matriz polimérica. A análise espectral indicou que as absorbâncias específicas das amostras GLM e GAL apresentaram uma grande semelhança nos seus espectros de IV, o que mostra que as absorbâncias específicas dos grupos funcionais da GAL na superfície das micropartículas têm quase as mesmas características químicas da GAL pura (Figura 4). As 
frequências de absorções estão de acordo com as estruturas típicas de galactomanana de $D$. regia e de outras espécies. ${ }^{17}$ Por meio da análise dos espectros percebe-se que não houve interação química entre a alantoína e polímero, uma vez que não há o surgimento de novas bandas de absorção, o que é útil nos processos de microencapsulação, denotando que a galactomanana de D. Régia se apresenta como um bom carreador de bioativos, atóxico e sustentável.

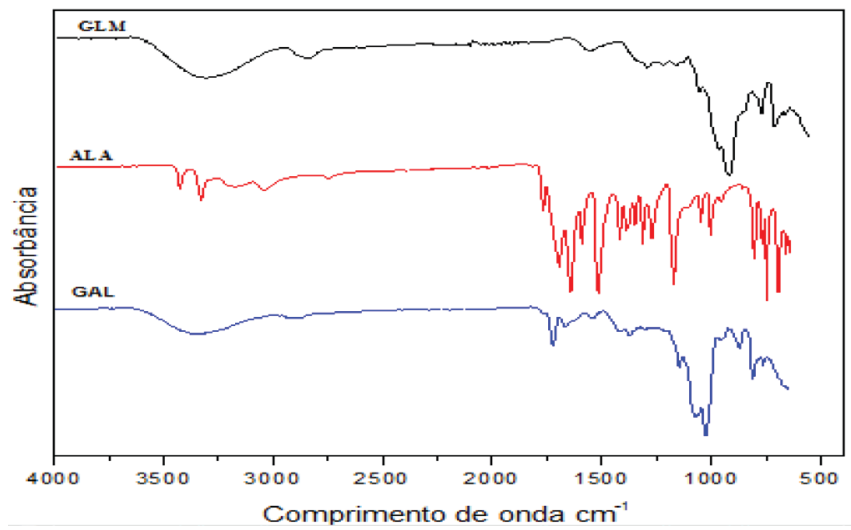

Figura 4. Espectro do IV da galactomanana (GAL), alantoína (ALA) e das micropartículas de galactomanana/alantoína (GLM)

\section{Microscopia eletrônica de varredura (MEV)}

A imagem MEV da alantoína (ALA) indica a presença de cristais de forma não definida (similar a um elipsoide). A micrografia da galactomanana (GAL) revela partículas de aparência esférica dispostas de forma aleatória, com variação de tamanho e espessura. Enquanto que, a imagem das micropartículas de galactomanana/ alantoína(GLM) revelam um formato regular, permitindo ainda uma boa indicação de tamanho (tamanho médio de 2,84 $\pm 0,41 \mu \mathrm{m}$ ). Também foi observado que a superfície das microcápsulas esféricas se mostrou lisa e regular (Figura 5).

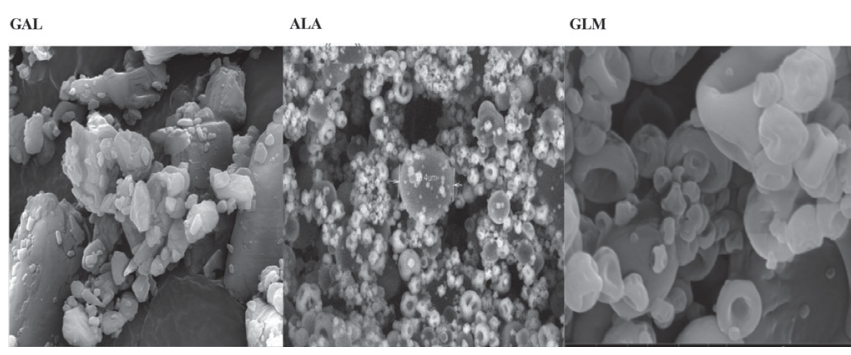

Figura 5. Micrografia da galactomanana (GAL), alantoína (ALA) e micropartículas de galactomanana/alantoína (GLM)

A morfologia da superfície de sistemas poliméricos tem um papel importante no seu processo de degradação e de liberação dos fármacos neles presentes, pois a presença de poros ou canais na matriz pode permitir uma difusão do fármaco possivelmente não controlada pela velocidade de degradação do polímero. A quase ausência de cristais adsorvidos na superfície das microcápsulas esféricas de galactomanana/alantoína (GLM) sugere uma boa e efetiva encapsulação do princípio ativo. Embora a concentração teórica seja de 10\%, raros são os casos em que $100 \%$ do fármaco é efetivamente encapsulado durante a formação de microesferas poliméricas, podemos considerar uma eficiência de encapsulamento (EE) bastante satisfatória, uma vez que apresentou $84 \%$ do ativo incorporado ao polímero, o que está de acordo com a literatura, cujos dados demostram uma eficiência de encapsulação de $86 \%$ para bixina encapsulada em goma arábica/sacarose (95:5), 82,2\% de EE para o licopeno encapsulado em gelatina/sacarose (3:7), 90,2\% de EE para a bixina em caseinato de sódio e 55,9 $\pm 5,6 \%$ de EE para a vitamina $\mathrm{B}_{2}$ encapsulada com nanopartículas de alginato/quitosana. ${ }^{18-21}$

Vale ressaltar que a literatura comercial relata uma concentração de 0,2 a $2 \%$ de alatoína pura em formulações tópicas, ${ }^{22}$ portanto, de acordo com os nossos estudos, seria necessário adicionar aproximadamente 2,4\% das micropartículas de alantoína/galactomanana em uma formulação tópica para obter o proporcional à $0,2 \%$ de alantoína pura mais estável e de liberação gradual.

\section{Análises térmicas}

O termograma DSC da alantoína mostrou um pico forte endotérmico de $230{ }^{\circ} \mathrm{C}$ na temperatura correspondente ao seu ponto de fusão, o que explica a sua cristalinidade. Já o termograma DSC da galactomanana apresenta picos típicos de polissacarídeo natural com eventos endotérmicos na faixa de temperatura entre $150{ }^{\circ} \mathrm{C}$ e 1175 ${ }^{\circ} \mathrm{C}$, aproximadamente, o que pode ser explicado pela evaporação da água. Resultados semelhantes foram observados em outras galactomananas. ${ }^{23} \mathrm{~A}$ ausência do pico endotérmico forte da ALA nas micropartículas de GLM atribuído a temperatura de fusão da região cristalina é sugestivo da perda da sua estrutura cristalina original resultante do processo de encapsulamento. A ausência do pico de fusão de ALA é também um indicativo de que a alantoína pode ter sido completamente encapsulada (Figura 6), o que está de acordo com a literatura, cujos dados revelam um comportamento similar no desenvolvimento de micropartículas de aciclovir com quitosana, no microencapsulamento da hesperidina e ao encapsular a vitamina C..$^{24,25}$

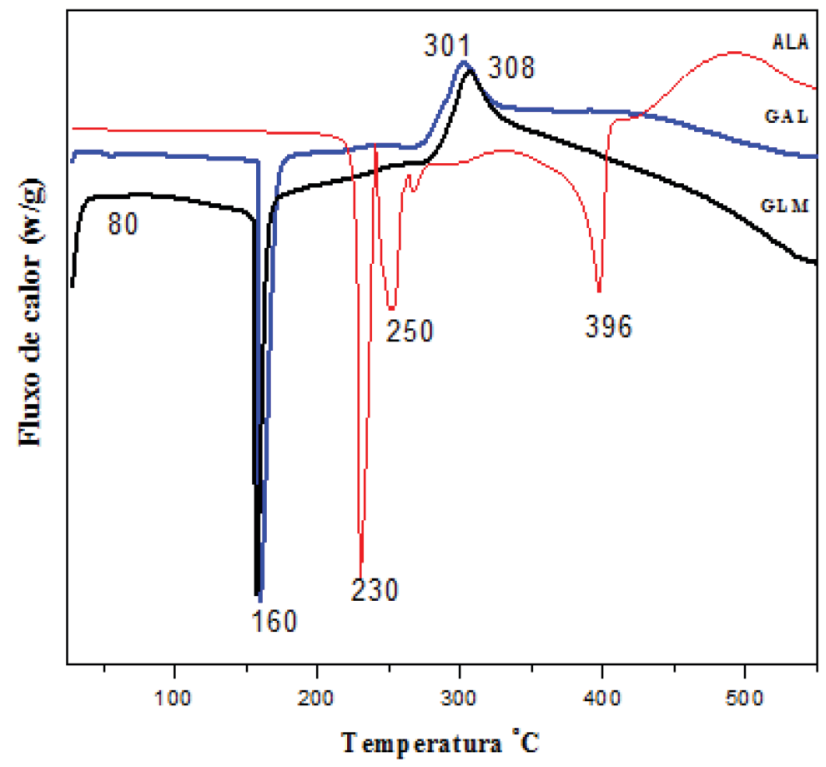

Figura 6. Termogramas de DSC da galactomanana (GAL), alantoína (ALA) e micropartículas de galactomanana/alantoína (GLM)

Conforme o perfil de degradação da amostra de galactomanana (GAL), dois eventos característicos de perda de massa foram observados. O primeiro, em torno de $50{ }^{\circ} \mathrm{C}$, apresentou $11,50 \%$ de perda de massa e pode ser explicado pela desidratação da amostra bem como água adsorvida na superfície da amostra. O segundo evento, próximo a $300{ }^{\circ} \mathrm{C}$, exibiu perda de massa por volta de $82,44 \%$ e pode ser atribuído à degradação do polissacarídeo. ${ }^{26}$

Já o TGA no perfil das micropartículas de galactomanana/alantoína sob ação da temperatura, a maior perda de massa dos compostos ocorre pouco depois do processo de fusão, em torno de $250-330^{\circ} \mathrm{C}$. Comparando as curvas obtidas para galactomanana e micropartículas de galactomanana/alantoína observa-se que não foram detectadas 
mudanças significativas no valor do termograma. Observa-se, ainda, que as amostras foram estáveis na faixa de $15-230{ }^{\circ} \mathrm{C}$ e tiveram perda significativa de massa na faixa de $250-330^{\circ} \mathrm{C}$ (Figura 7).
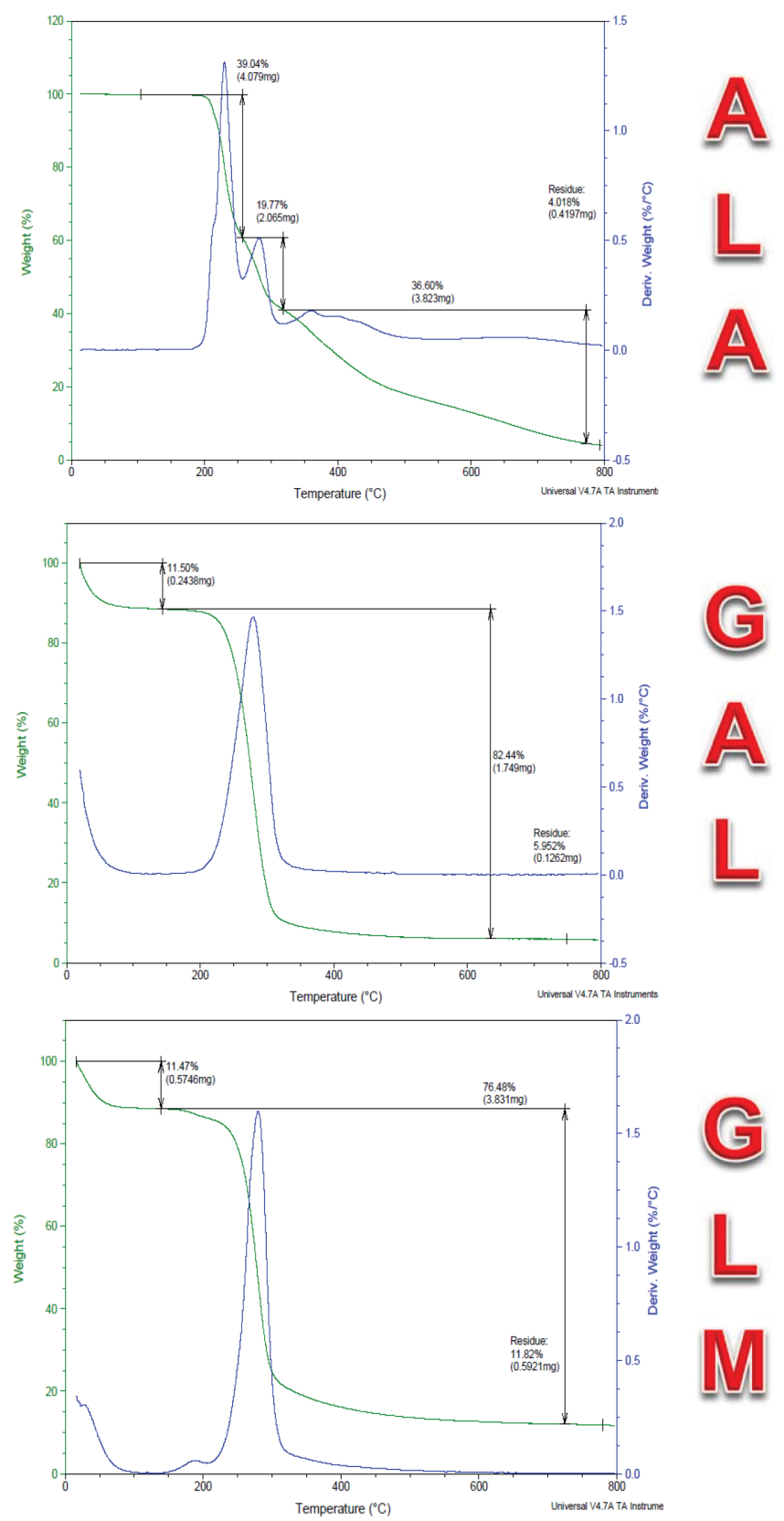

Figura 7. Perfil de degradação comparativo das amostras de alantoína (ALA), galactomanana (GAL) e micropartículas de galactomanana/alantoína (GLM)

\section{Difração de Raios- $X(D R X)$}

$\mathrm{Na}$ análise do DRX da galactomanana (GAL), observa-se picos largos não definidos, o que mostra que esta substância não apresenta cristais, portanto possui estrutura amorfa. ${ }^{27} \mathrm{O}$ estado cristalino da alantoína (ALA) foi evidenciado no difratograma de DRX; o perfil exibiu vários picos bem definidos em $2 \theta$. O difratograma das micropartículas de galactomanana/alantoína (GLM) apresentou picos semelhantes do biopolímero puro, indicando que o fármaco pode estar encapsulado no sistema, na forma amorfa (Figura 8).

Comportamento semelhante foi observado no trabalho de Devkar e colaboradores, ${ }^{28}$ os quais produziram carreadores nanoestruturados por freeze dried e também observaram que houve redução da intensidade dos picos de DRX da droga, sugerindo a conversão da forma cristalina para a forma amorfa.

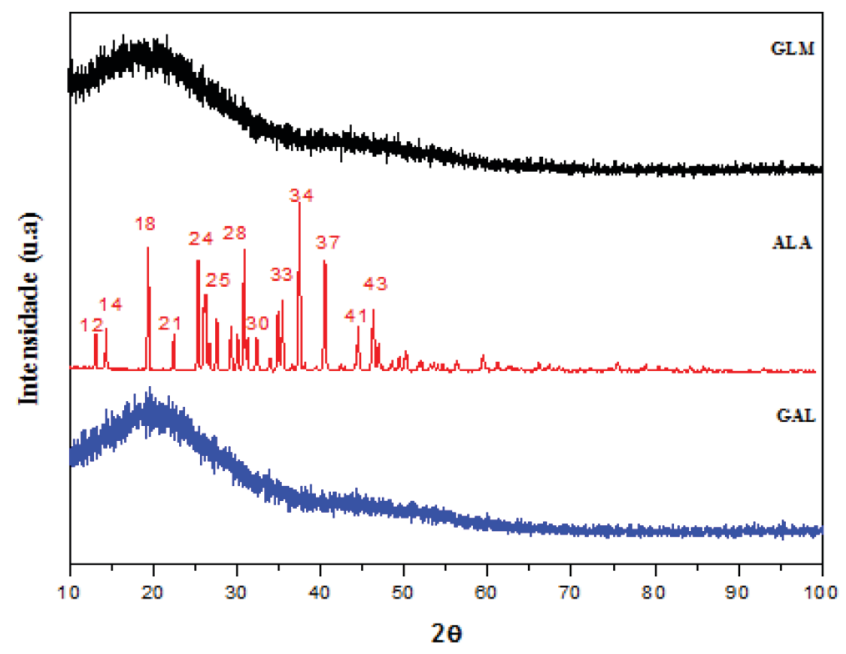

Figura 8. Difração de raios-x da galactomanana $(G A L)$, alantoína $(A L A)$ e microcápsulas galactomanana/alantoína (GLM)

\section{Ensaio de liberação in vitro com dispersão direta no meio}

A velocidade de liberação de fármacos a partir de sistemas microparticulados pode ser influenciada pela combinação de diversos fatores, como características da matriz polimérica (espessura, porosidade, capacidade de intumescimento), tamanho das micropartículas, localização do ativo no interior das partículas, das propriedades físico-químicas do fármaco e do polímero. ${ }^{29}$

Os resultados apresentados demonstraram que o ensaio feito com as micropartículas galactomanana/alantoína configuraram uma liberação controlada, uma vez que os desvios-padrões obtidos foram valores bem abaixo dos valores que a Farmacopeia Brasileira preconiza que é correspondente a $5 \% .{ }^{29} \mathrm{Com}$ estes resultados, pode-se inferir que as amostras de micropartículas galactomanana/alantoína foram bem elaboradas e, além da liberação gradual, tem a vantagem de aumentar o tempo de estabilidade da alantoína. Outro aspecto relevante que se deve inferir com estes resultados é que, ao final das 6 horas do ensaio de liberação, o teor final de liberação chegou aos $100 \%$, valor este que está de acordo com o intervalo estipulado e permitido pela Farmacopeia Brasileira, os quais podem variar em $85 \%$ a $120 \% .{ }^{30}$ Observa-se, ainda, que ocorreu a liberação controlada do ativo alantoína através da parede polimérica das micropartículas, ao longo das 6 horas de ensaio, pois a mesma liberou este ativo de forma bastante gradual. Já o ensaio das amostras de alantoína pura chegou à $100 \%$ em até 5 minutos (Figura 9).

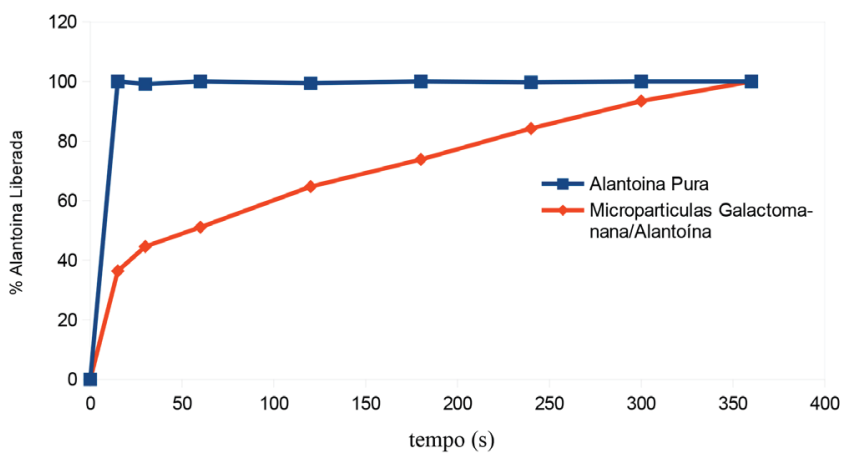

Figura 9. Liberação da alantoína na formulação teste (micropartículas de galactomanana/alantoína) X Liberação da alantoína pura 
Considerando tudo o que foi apresentado acima, pode-se concluir que a produção destas micropartículas, além de promover a proteção do ativo, aumentando sua estabilidade e vida útil, também permite que este material possa ser administrado como fármaco, uma vez que a microencapsulação ajudou a controlar a liberação da alantoina.

\section{CONCLUSÕES}

A produção de microcápsulas de alantoína revestidas com galactomanana extraída da espécie $D$. regia através da técnica de secagem por atomização por spray drying, juntamente com a caracterização físico-química, a eficiência de encapsulação e a liberação controlada foram relevantes para o composto alantoína. As informações obtidas no espectro de IV sugeriram a formação da parede polimérica da cápsula contendo alantoína. As micropartículas apresentaram morfologia com intervalo de tamanho de partícula média 2,84 \pm $0,41 \mu \mathrm{m}$, observadas através da análise do MEV. A difração de raios $\mathrm{X}$ mostrou a perda de cristalinidade do ativo após a encapsulação e a calorimetria explanatória diferencial exibiu a perda do seu pico de fusão. A eficiência de encapsulação para as micropartículas foi de aproximadamente $84 \%$ para as diferentes condições ambientais, como temperaturas de $25{ }^{\circ} \mathrm{C}$ e $60{ }^{\circ} \mathrm{C}$, e sob ação da luz $(\lambda)$. A liberação controlada das microcápsulas e somente do ativo alantoína apresentaram os resultados de acordo com a Farmacopeia Brasileira 2010, mostrando-se uma liberação gradual da alantoína presente nas micropartículas ao longo do tempo estipulado para analise em comparação a liberação do ativo puro, a qual foi muito rápida. Este resultado permitiu inferir que a produção destas micropartículas, além de promover a proteção do ativo, aumentando sua estabilidade e vida útil, também permite que este material possa ser administrado como fármaco de uso tópico em pacientes que necessitem tratamento de regeneração celular cutânea. O conjunto dos resultados obtidos neste trabalho é relevante e credencia o sistema desenvolvido como uma possível alternativa para formação de fármacos naturais encapsulados.

\section{AGRADECIMENTOS}

À FUNCAP, ao CNPq e à CAPES pelas bolsas e apoio financeiro, ao Mestrado Acadêmico em Recursos Naturais da UECE, ao Laboratório de Polímeros e Inovação da UFC, ao Laboratório de Química de Produtos Naturais da UECE, ao CEAUREM, à Central Analítica da UFC, ao Departamento de Física da UFC, ao Laboratório de Controle de Qualidade da Farmácia Escola da UFC e à Central Analítica da UFBA.

\section{REFERÊNCIAS}

1. Oréfice, R. L.; Pereira, M. M.; Mansur, H. S.; Biomateriais: fundamentos e aplicações, $1^{\mathrm{a}}$ ed., Guanabara Koogan: São Paulo, 2005.

2. Villanova, J. C. O.; Oréfice, R. L.; Polímeros: Ciência e Tecnologia. 2010, 20, 51 .

3. Aulton, M. E.; Delineamento de formas farmacêuticas, $2^{\mathrm{a}}$ ed., Artmed: Porto Alegre, 2005.

4. Swarbrick, J.; Encyclopedia of Pharmaceutical Technology, 30a ed., Informa Healthcare: England, 2007.
5. Lafferty, M. C.; E.; Hendry, C.; Alistair, F.; Nursing Standard 2012, 27, 35.

6. Chen, Y. E.; Tsao, H.; J. Am. Acad. Dermatol. 2013, 69, 143.

7. Greaves, N. S.; Ashcroft, K. J.; Baguneid, M.; Bayat, A.; J. Dermatol. Sci. 2013, 72, 206.

8. Pyun, D. O. G.; Choi, H. J.; Yoon, H. S.; Thambi, T.; Lee, D. S.; Colloids Surf., B 2015, 135, 699.

9. Gurtner, G. C.; Werner, S.; Barrandon, Y.; Longaker, M. T.; Nature 2008, $453,314$.

10. Khalil, E. A.; Afifi, F. U.; Al-Hussaini, M.; J. Ethnopharmacol. 2007, 109, 104.

11. Ferrari, R.; Barbosa, A. M.; Ornelas, S. S.; Lano, M. E. D.; Barbosa, A. C. L.; Ensaios e Ciência: Ciências Biológicas, Agrárias e da Saúde 2012, 16, 227

12. Lyra, M. A. M.; Soares-Sobrinho, J. L.; Brasileiro, M. T.; La Roca, M. F.; Barraza, J. A.; Viana, O. S.; Rolim-Neto, P.; J. Lat. Am. J. Pharm. 2007, 26, 784

13. Moshfeghi, A. A.; Peyman, G. A.; Adv. Drug Delivery Rev. 2005, 57, 2047.

14. Vieira, Í. G. P.; Mendes, F. N. P.; Gallão, M. I.; Brito, E. S.; Food Chem. 2007, 101, 70 .

15. Moura, E. S.; Dissertação de mestrado, Universidade Federal de Minas Gerais, Brasil, 2005.

16. Souza, C. A. G.; Siqueira, S. M. C.; Amorim, A. F. V.; Morais, S. M.; Gonçalves, T.; Gomes, R. N.; Cunha, A. P.; Ricardo, N. M. P. S.; Quim. Nova 2015, 38, 877.

17. Siqueira, N. M.; Paiva, B.; Camassola, M.; Rosenthal-Kim, E. Q.; Garcia, K. C.; Dos Santos, F. P.; Soares, R. M.; Carbohydr. Polym. 2015, $133,8$.

18. Barbosa, M. I. M. J.; Borsarelli, C. D.; Mercadante, A. Z.; Food Res. Int. 2005, 38, 989 .

19. Shu, B.; Yu, W.; Zhao, Y.; Liu, X.; J. Food Eng. 2006, 76, 664.

20 Zhang, Y; Zhong, Q.; Food Hydrocolloids 2013, 33, 1.

21. Azevedo, M. A.; Bourbon, A. I.; Vicente, A. A.; Cerqueira, M. A.; Int. J. Biol. Macromol. 2014, 71, 141.

22. Fiedler, H. P.; Artzl. Kosmetol. 1985, 15, 87.

23. Souza, N. D. G.; Dissertação de mestrado, Universidade Federal do Ceará, Brasil, 2014.

24. Stulzer, H. K.; Tagliari, M. P.; Silva, M. A. S.; Laranjeira, M. C. M.; Lat. Am. J. Pharm. 2007, 26, 866.

25. Sansone, F.; Rossi, A.; Del Gaudio, P.; De Simone, F.; Aquino, R. P.; Lauro, M. R.; AAPS PharmSciTech 2009, 10, 391.

26. Sun, Y. C.; Wen, J. L.; Xu, F.; Sun, R. C.; Bioresour. Technol. 2011, 102, 5947.

27. Lima, D. U.; Oliveira, R. C.; Buckeridge, M. S.; Carbohyd Polym. 2006, 52,36727

28. Devkar, T. B.; Tekade, A. R.; Khandelwal, K. R.; Colloid Surf., B 2014, 122, 143.

29. Lopes, C. M.; Lobo, J. M. S.; Costa, P.; Braz. J. Pharm. Sci. 2005, 41, 143.

30. Agência Nacional de Vigilância Sanitária; Farmacopeia Brasileira, Editora Fiocruz: Brasília, Brasil, 2010. 